\title{
Neuroprotection of lamotrigine on hypoxic-ischemic brain damage in neonatal rats: Relations to administration time and doses
}

\author{
Yong-Hong $\mathrm{Yi}^{\prime}$ \\ Wen-Chao Guo' \\ Wei-Wen Sun' \\ Tao Su' \\ Han Lin' \\ Sheng-Qiang Chen' \\ Wen-Yi Deng' \\ Wei Zhou ${ }^{2}$ \\ Wei-Ping Liao' \\ 'Department of Neurology, Institute \\ of Neurosciences and the Second \\ Affiliated Hospital, ${ }^{2}$ Department of \\ Neonatology, Affiliated Guangzhou \\ Children's Hospital, Guangzhou \\ Medical College, Guangzhou, \\ Guangdong Province, P.R. China
}

\begin{abstract}
Lamotrigine (LTG), an antiepileptic drug, has been shown to be able to improve cerebral ischemic damage by limiting the presynaptic release of glutamate. The present study investigated further the neuroprotective effect of LTG on hypoxic-ischemic brain damage (HIBD) in neonatal rats and its relations to administration time and doses. The HIBD model was produced in 7-days old SD rats by left common carotid artery ligation followed by $2 \mathrm{~h}$ hypoxic exposure ( $8 \%$ oxygen). LTG was administered intraperitoneally with the doses of 5 , 10,20 , and $40 \mathrm{mg} / \mathrm{kg} 3 \mathrm{~h}$ after operation and the dose of $20 \mathrm{mg} / \mathrm{kg} 1 \mathrm{~h}$ before and $3 \mathrm{~h}, 6 \mathrm{~h}$ after operation. Blood and brain were sampled $24 \mathrm{~h}$ after operation. Nissl staining, terminal deoxynucleotidyl transferase biotin-dUTP nick end labeling (TUNEL), and neuron-specific enolase (NSE) immunohistochemical staining were used for morphological studies. Water content in left cortex and NSE concentration in serum were determined. LTG significantly reduced water content in the cerebral cortex, as well as the number of TUNEL staining neurons in the dentate gyrus and cortex in hypoxic-ischemia (HI) model. Furthermore, LTG significantly decreased the NSE level in serum and increased the number of NSE staining neurons in the cortex. These effects, except that on water content, were dose-dependent and were more remarkable in the pre-treated group than in the post-treated groups. These results demonstrate that LTG may have a neuroprotective effect on acute HIBD in neonates. The effect is more prominent when administrated with higher doses and before HI.
\end{abstract}

Keywords: hypoxic-ischemic brain damage, lamotrigine, neonatal rat, neuroprotection

\section{Introduction}

Hypoxic ischemia (HI) is a common cause of brain damage in fetus and neonates. It is believed that HI may cause brain damages by activating a cascade of biochemical events, in which glutamate receptor plays an important role in triggering the toxic events (Hattori and Wasterlain 1990; Strijbos et al 1996). Previous studies have shown that the antagonists of $N$-methyl-D-aspartate (NMDA) and $\alpha$-amino-3-hydroxy-5-methylisoxazole4-propionic acid (AMPA) receptor have a neuroprotective effect on hypoxic-ischemic brain damage (HIBD) in stroke models (Nellgard and Wieloch 1992; Scatton et al 1994). However, the adverse profile of glutamate receptor antagonists limits their application in clinical practice (Scatton et al 1994). An alternative approach to ameliorate ischemic damage is to inhibit the presynaptic release of glutamate. Lamotrigine (LTG), a new kind of anticonvulsant drug with a action of inhibiting excessive presynaptic release of glutamate (Leach et al 1986) by blocking voltage-sensitive sodium channels (Cheung et al 1992; Lees and Leach 1993), has been demonstrated to be effective for HIBD in focal and global cerebral ischemia model (Graham et al 1993; Smith and Meldumr 1995; Wiard et al 1995; Crumrine et al 1997), as well as in the in vitro experiments (Calabresi et al 2003). Although the neuroprotective effects of LTG have been well demonstrated 
in ischemic models with adult rats and LTG has even been used as combination therapy for the patients with ischemic stroke (Chen et al 2002), little is known about its effects on neonatal HIBD (Papazisis et al 2007). Since the immature brain is highly sensitive to glutamate excitotoxicity (Barks and Silverstein 1992; Scatton et al 1994; Puka-Sundvall et al 1997), it is conceivable that LTG might be useful for HIBD in neonates. Recent reports have shown that LTG has the ability of extensive placental transfer and also a good tolerability in infants (Ohman et al 2000; Mikati et al 2002), provided a basis for the clinical application in neonates or infants. The aim of the present study is to investigate the possible neuroprotective effects of LTG on HIBD in the perinatal period, and the relationships between the effect and administration time and doses. HI was induced according to the protocol in a widely used animal model of perinatal asphyxia (Rice and Vannucci 1981). Histological and biochemical changes were examined to evaluate the damage of HI. Neuron-specific enolase (NSE), an isoenzyme of the glycolytic enzyme enolase (2-phosphoD-glycerate hydrolase), was adopted as one of important quantitative index in our study, because it was reported that NSE is released into the cerebral spinal fluid (CSF) and systemic circulation when neuronal damage has occurred (Schoerkhuber et al 1990; Martens et al 1998; Wang et al 1999; Verdu et al 2001), and NSE is highly specific to the neuron.

\section{Material and methods HIBD model}

All experiments were performed in accordance with the institutional animal care guidelines. Common carotid artery occlusion combined with hypoxia in postnatal day 7 (P7) rat pups was performed to induce HIBD (Rice and Vannucci 1981). Rat pups were placed in an incubator with ambient temperature of $37^{\circ} \mathrm{C}$. Under deep ether anesthesia, the left common carotid artery was isolated, double ligated, and cut between the ligatures. After the surgical procedure, the animal was put to recover for $2 \mathrm{~h}$ in the temperature-controlled incubator and was then exposed to hypoxia for $2 \mathrm{~h}$ : placed in an enclosed and vented chamber that was partially submerged in water $\left(37^{\circ} \mathrm{C}\right)$. Hypoxia was induced by continuous flow of warmed, humidified gas with $8 \%$ oxygen that was balanced with $92 \%$ nitrogen at the flow rate of $0.5 \mathrm{~L} / \mathrm{ml}$. All $\mathrm{HI}$ animals showed symptoms of hemiplegia and anoxia.

\section{Study design}

In order to evaluate the effects of different doses, $\mathrm{P} 7$ rats were divided into 4 different dose groups, ie, $5,10,20$, and $40 \mathrm{mg} / \mathrm{kg}$ ( $n=14$ for each dose group). LTG was given intraperitoneally at $3 \mathrm{~h}$ after the ischemia. The dose of $20 \mathrm{mg} / \mathrm{kg}$ was selected to determine the therapeutic time window, which was administrated intraperitoneally $1 \mathrm{~h}$ before (pre-treated), or $3 \mathrm{~h}, 6 \mathrm{~h}$ after the ischemia (post-treated) $(\mathrm{n}=14$ for each time point group). The dose range was derived from the previous report (Smith and Meldumr 1995; Wiard et al 1995; Lee et al 2000) and from our preliminary experiments. Since a pharmacokinetic study in rats has shown that LTG was rapidly absorbed following the intraperitoneal injection with a peak concentration achieved at $0.2-1.0 \mathrm{~h}$ for $20 \mathrm{mg} / \mathrm{kg}$ (Walker et al 2000), LTG was administered $1 \mathrm{~h}$ before ischemia to achieve a stable plasma concentration in the pre-treated group. It has been reported that LTG plasma elimination half-life is approximately $28 \mathrm{~h}$ in Wistar rat with $10 \mathrm{mg} / \mathrm{kg}$ intraperitoneally (Castel-Branco et al 2005), so a single dose administration was adopted in our study. Pure LTG (Glaxo Wellcome Co.) was suspended in $0.5 \%$ methylcellulose $(1 \mathrm{mg} / \mathrm{ml})$ before the experiment. Sham-operated controls (sham group, $n=14$ ) and hypoxic-ischemic controls (HI group, $n=14$ ) were only given equal volume of methylcellulose $3 \mathrm{~h}$ after HI. Six of 14 rats in each group were used for neuropathological examination, including immunohistochemical staining of NSE, terminal deoxynucleotidyl transferase biotin-dUTP nick end labeling (TUNEL) and Nissl staining. And 8 from each treatment group were used for determination of water content and serum NSE level.

\section{Measurement of water content}

A rat was anesthetized with chloral hydrate $(350 \mathrm{mg} / \mathrm{kg})$ $24 \mathrm{~h}$ after HI, and the brain was rapidly excised. The sample of cortex from left cerebral hemisphere (ipsilateral to common carotid artery ligation) was weighed in milligram unit. The specimen was subsequently desiccated at $110^{\circ} \mathrm{C}$ for $24 \mathrm{~h}$. Reweighing the dry weight of the tissue, the water content was obtained by subtraction from the wet weight [water content $=($ wet weight- dry weight $) /$ wet weight $\times 100 \%$ ]

\section{Determination of neuron-specific enolase (NSE) in serum}

Blood was collected during excision. Blood samples were left to clot at room temperature for 20-30 $\mathrm{min}$ and then centrifuged and frozen at $-18{ }^{\circ} \mathrm{C}$ until assayed. The concentration of NSE in serum was measured by enzyme immunoassay NSE kit (Canag diagnostics AB Company, Denmark).

\section{Neuropathology}

$24 \mathrm{~h}$ after $\mathrm{HI}$, the rat was anesthetized with $10 \%$ chloral hydrate $(0.35 \mathrm{ml} / 100 \mathrm{~g}$, ip) and perfused transcardially 


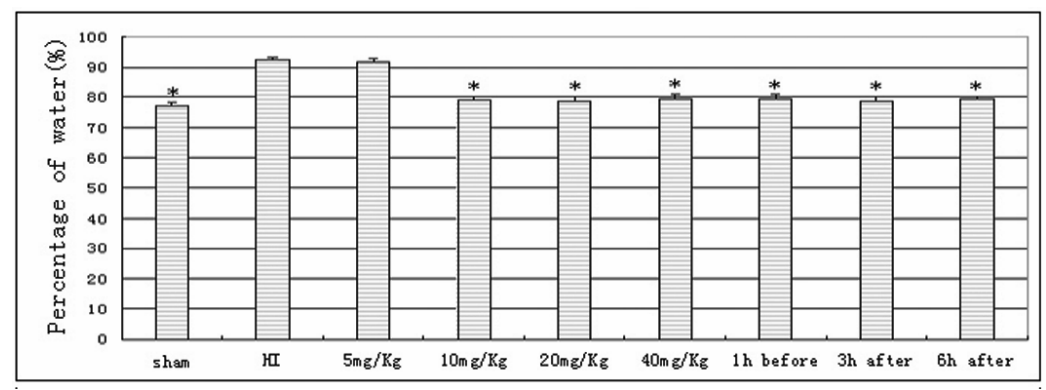

Figure I Changes in the water content in the cortex of ischemic hemisphere. The asterisks indicate statistically significant differences vs $\mathrm{HI}$ group value $(* \mathrm{P}<0.0 \mathrm{I}, \mathrm{n}=8 \mathrm{for}$ each group). LTG treatment doses and the time points of administration are indicated in horizontal axis.

with $10-15 \mathrm{ml}$ of saline $(0.9 \% \mathrm{NaCl})$, followed by 20-30 $\mathrm{ml}$ of $4 \%$ paraformaldehyde in $0.01 \mathrm{M}$ PBS (pH 7.4). The brain was excised and postfixed for $24 \mathrm{~h}$ at $4{ }^{\circ} \mathrm{C}$, then through $10 \%, 20 \%$, and $30 \%$ sucroses for cytoprotection. OCT-embedded tissue was frozen at $-80^{\circ} \mathrm{C}$ and sectioned on a cryotome at a thickness of $30 \mu \mathrm{m}$ along the coronal plane. The sections from B-2.2 to B-4.2 were selected. The adjacent sections were processed for either immunohistochemical staining of NSE or TUNEL and Nissl staining. NSE staining neurons in cortex and TUNEL positive cells in the cortex and dentate gyrus were quantified by a single observer who had no knowledge of the treatment received by the animals using a light microscope $(400 \times)$. The mean was derived from six counts (three sections from each sample and two areas from each section) in each animal for statistical analysis.

\section{Statistical analysis}

Data are expressed as mean \pm SE. Data were analysed using the ANOVA test followed by post-hoc multiple comparisons by Dunnett's test.

\section{Results}

\section{The effect of LTG on brain edema}

Hypoxic ischemia resulted in a significant increase of the water content. The treatments of $\mathrm{LTG} \geqq 10 \mathrm{mg} / \mathrm{kg}$ reduced water contents in $\mathrm{HI}$ animals significantly $(P<0.01)$. No significant difference in water content was detected between the each dose groups with LTG higher than $10 \mathrm{mg} / \mathrm{kg}$, nor between the pre-treated and the post-treated groups (Figure 1).

\section{The effect of LTG on neuron necrosis and apoptosis}

Nissl's staining exhibited extensive brain damage in the hemisphere that subjected to hypoxic-ischemic injury. The regular architecture was destroyed with a marked loss of Nissl staining. Multiple necroses with shrinkage of cell body, pyknosis of nucleus, loss of Nissl substance, and disappearance of nucleolus, were found in the cortex, hippocampus, striatum, and thalamus. The impairment was reduced in the treated groups with LTG dose higher than $20 \mathrm{mg} / \mathrm{kg}$ (Figure 2).

TUNEL positive cells appeared in many regions of the ischemic hemisphere including frontal lobe, parietal lobe, callosal gyrus, striatum and hippocampus, particularly in the pyramidal layer of CA1 region and the granular cell layer of dentate gyrus in the hippocampus (Figure 3). Most of the TUNEL positive cells had the features of apoptosis with typical demilune and apoptotic bodies. The numbers of TUNEL positive cells in the cortex and dentate gyrus in the ischemic hemisphere were significantly decreased in the LTG-treated groups with doses $\geqq 10 \mathrm{mg} / \mathrm{kg}(P<0.01)$. There were significant differences between each dose groups with LTG of 10, 20, and $40 \mathrm{mg} / \mathrm{kg}$. Significant differences were also found between the pre-treated and the post-treated groups $(P<0.01)$, but not between the $3 \mathrm{~h}$ post-treated and the $6 \mathrm{~h}$ post-treated groups (Figure 4 ).

\section{The effect of LTG on NSE level in serum and the number of NSE staining neurons}

In HI group, NSE staining cells were rarely observed in the cerebral cortex and statistic analysis showed a significant decrease in cell count (Figure 5). The NSE level in serum was significantly increased in HI group. The treatment of LTG $\geqq 20$ $\mathrm{mg} / \mathrm{kg}$ resulted in a significant increase of NSE staining cells in the cortex and a decrease of NSE level in serum $(P<0.01)$ (Figure 6). These changes were more significant in the pretreated group than in the post-treated groups $(P<0.01)$. There was no significant difference between the $3 \mathrm{~h}$ post-treated and the $6 \mathrm{~h}$ post-treated groups.

\section{Discussion}

The neuroprotective effect of LTG on HIBD in neonatal model has recently been reported (Papazisiset al 2007). 


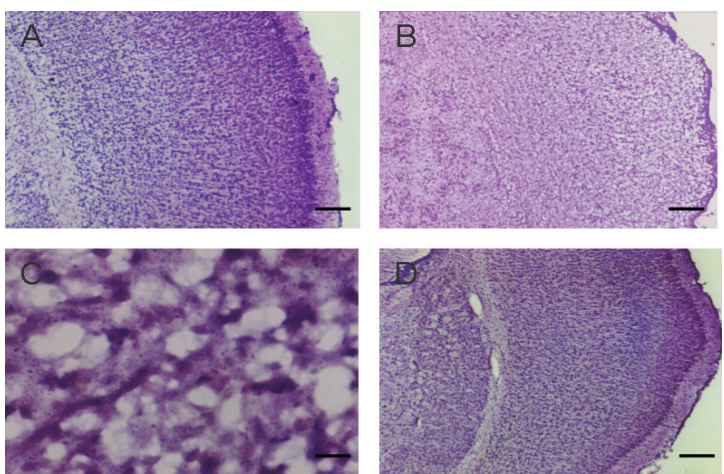

Figure 2 Photomicrographs of Nissl's staining of the left cerebral cortex. A. A normal imaging from a sham-operated control. B. The regular architecture was destructed in the HI cortex. C. A high power view of the HI cortex, which shows necrosis of the cells with shrinkage of the cell body, pyknosis of nucleus, loss of Nissl substance, and disappearance of nucleolus. D. An imaging of the cortex from a subject received LTG of $40 \mathrm{mg} / \mathrm{kg}, 3 \mathrm{~h}$ after H. Scale bar $=250 \mu \mathrm{m}$ in A, B and D, and $25 \mu \mathrm{m}$ in C.

Further to our preliminary study (Yi et al 2003), the present data demonstrate that LTG has a neuroprotective effect on HIBD in neonatal rats, shown by reducing the brain edema, protecting the neurons from necrosis and apoptosis, and inhabiting the leakage of NSE from the neurons. Results from the present study provided some evidence of neuroprotection of LTG for neonatal HIBD.

The dose-efficacy relationship on the neuroprotection of LGT has not well defined in previous studies, although there is a report from an in vitro experiment (Wang et al 2001). In order to study the dose-efficacy relationship and to know the effective dose of LTG for possible clinical application, we tried to quantify HIBD by measuring the water content in the cortex, TUNEL, and NSE staining cells in the brain, and NSE in serum. Brain edema is one of the basic pathological changes during HI. It is shown that LTG of more than $10 \mathrm{mg} / \mathrm{kg}$ can significantly decrease the water content in the HI cortex. However, we failed to find a difference between each dose, which might be imputed to the narrow range (77.2\%-92.4\%) of changes in water content, which is not sensitive enough to show minor alternations.

A good correlation between dose and efficacy is demonstrated in the dose range of $5-40 \mathrm{mg} / \mathrm{kg}$, and the effective dose is from $10 \mathrm{mg} / \mathrm{kg}$, shown by the TUNEL cell number. The correlation dose range is $10-40 \mathrm{mg} / \mathrm{kg}$ and the effective dose is from $20 \mathrm{mg} / \mathrm{kg}$, shown by both the NSE staining cell number and the NSE concentrations in serum. The previous report has demonstrated a good relationship between the neurological outcome and serum NSE levels in the patients after cardiac arrest (Schoerkhuber et al 1990), suggesting that serum NSE levels can reflect the degrees of brain injury. The increase level of NSE in serum has been commonly accepted to be as a maker of the neuronal damage. Our results show an elevated NSE level in serum with decreased NSE staining in the neurons in neonatal rats after HI, confirmed that ischemia may cause the leakage of cytosolic brain enzymes into the blood and the elevation of NSE in serum can reflect the degree of brain damage. It is clinical significant because only blood sample can be obtained in practice. The effective dose rang of $10-40 \mathrm{mg} / \mathrm{kg}$ in the present study covered the doses of $10-20 \mathrm{mg} / \mathrm{kg}$ that was reported previously (Smith and Meldumr 1995; Papazisis1 et al 2007). LTG of $10-40 \mathrm{mg} / \mathrm{kg}$ in rats is equivalent to $1.6-6.4 \mathrm{mg} / \mathrm{kg}$ in human, lower than the dose of $5-15 \mathrm{mg} / \mathrm{kg}$ for monotherapy in patients with epilepsy. Since LTG could be well tolerated within this dose range for partial seizures and infantile spasms in infants (Mikati et al 2002), it is reasonable to conceive that LTG could be used as a neuroprotective agent for HIBD in neonates clinically. The fact that LTG of higher doses within the range of $10-40 \mathrm{mg} / \mathrm{kg}$ is more effective in protecting neuronal damage, shown in the present study, suggests that a higher dose within tolerated limit could be adopted in practice.

The therapeutic time window is crucial for a neuroprotective agent, which is not well defined for LTG. Previous studies have selected different time points for observation, from $1 \mathrm{~h}$ before $\mathrm{HI}$ in histotoxic hypoxia model (Schulz et al 1995) to $5 \mathrm{~h}$ after recovery from cardiac arrest in rat (Crumrine et al 1997). The present results show that LTG given either before or after HI, may attenuate HIBD in neonatal rats, with better effect in the pre-treated group and equivalent effect in the groups treated $3 \mathrm{~h}$ and $6 \mathrm{~h}$ after HI.
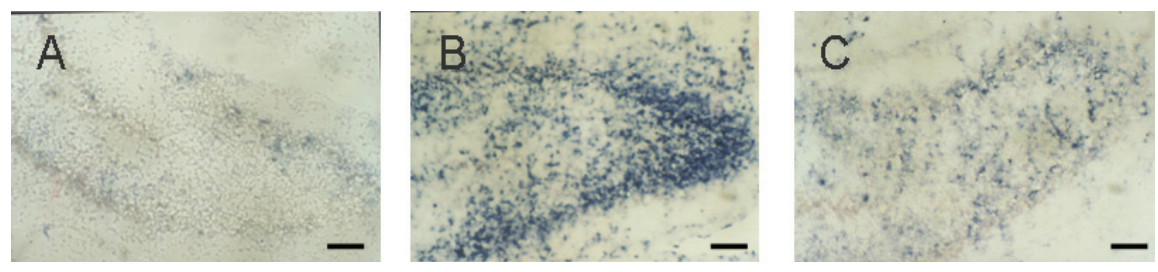

Figure 3 Photomicrographs of TUNEL staining of the dentate gyrus. A. Few TUNEL staining in the dentate gyrus from the sham-operated control. B. Marked TUNEL staining in the dentate gyrus in HI group. C. Reduced TUNEL staining in the dentate gyrus from the subject received LTG of $20 \mathrm{mg} / \mathrm{kg}, 3 \mathrm{~h}$ after HI. Scale bar $=250 \mu \mathrm{m}$. 


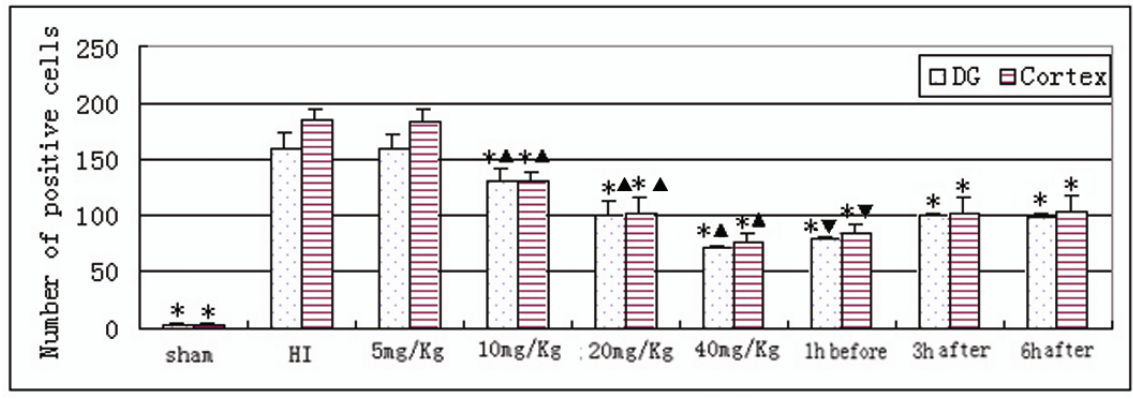

Figure 4 Comparison of the number of TUNEL staining cells in the cortex and dentate gyrus in different groups $(\mathrm{n}=6)$. *Indicates statistically significant differences vs HI group values $(P<0.0 \mathrm{I}){ }^{\wedge}$ Indicates statistically significant differences between each dose groups $(P<0.0 \mathrm{I})$. $^{\mathbf{V}}$ Indicates statistically significant difference between the pre-treated and post-treated groups $(P<0.01)$.
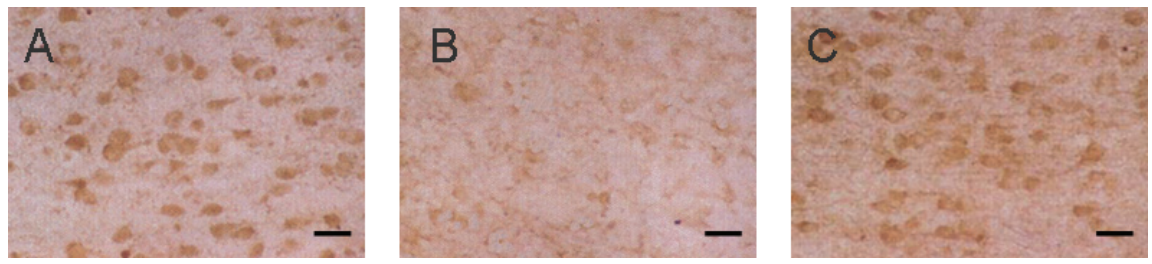

Figure 5 Photomicrographs of NSE immunohistochemical staining of the left cerebral cortex.A. Imaging shows the NSE staining neurons in the cortex from the sham-operated control. B. Reduced NSE staining in HI group. C. Imaging shows the NSE staining neurons in the cortex from the subject received LTG of $20 \mathrm{mg} / \mathrm{kg}, 3 \mathrm{~h}$ after $\mathrm{HI}$. Scale bar $=50 \mu \mathrm{m}$.

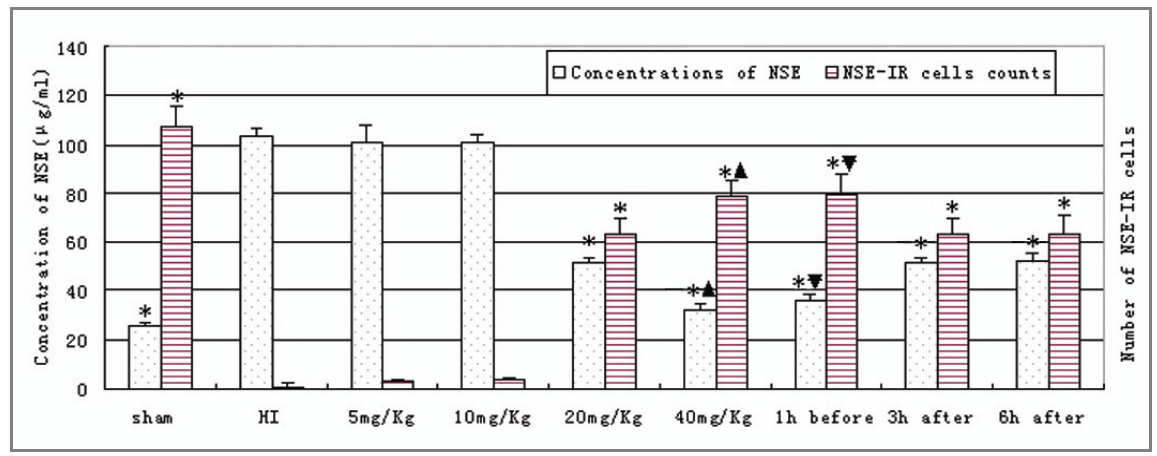

Figure 6 Alternations of the NSE level in serum $(n=8)$ and the number of NSE staining cells in the cortex $(n=6)$ in different groups. *Indicates statistically significant differences vs $\mathrm{HI}$ group values $(P<0.0 \mathrm{I})$. NSE level in serum is decreased, whereas the number of NSE staining cells is increased, comparing with $\mathrm{HI}$ controls. $\mathbf{\Delta}$ Indicates statistically significant differences between LTG treated-groups of $20 \mathrm{mg} / \mathrm{kg}$ and $40 \mathrm{mg} / \mathrm{kg}(P<0.0 \mathrm{I}){ }^{\nabla}$ Indicates a statistically significant difference between the pre-treated and post-treated groups $(P<0.01)$.

Extended time points are needed to define the exact time window in further studies.

Clinically, neuroprotective agents may be used either in patient known at the risk of hypoxic-ischemic brain injury or as post-insult "rescue" therapy. HIBD in neonatal occurs most frequently in the antepartum or intrapartum periods during which the drug administration is usually difficult. Therefore, the essential characteristics for a neuroprotective agent in prevention and treatment of neonatal HIBD should include: First of all, a wide therapeutic time window; secondly, the ability to reach peak concentration in the brain rapidly after administration; thirdly, it can pass through the placenta when it is given during antepartum or intrapartum periods.
The present study has shown that LTG has a therapeutic time window of at least $6 \mathrm{~h}$ after hypoxic-ischemia. It is demonstrated that LTG can be detected in the CSF $10 \mathrm{~min}$ after intraperitoneal administration and the penetration halftime into brain extracellular fluid is $0.51 \pm 0.11 \mathrm{~h}$ (Walker et al 2000). It has been reported that the maternal plasma LTG concentration at delivery is similar to that from the umbilical cord, and the plasma concentration in newborn declined slowly after then (Ohman et al 2000; Mikati et al 2002). Together, these data suggest that LTG has the potential for use as a neuroprotective agent in acute $\mathrm{HI}$ in neonates, as well as in pregnant women known at the risk of perinatal asphyxia. 
In summary, the present results demonstrated that LTG of tolerable doses initiated either before or after HI can attenuate the brain damage in $\mathrm{P} 7$ rats, suggesting the potential for clinical use in prevention and treatment of HIBD.

\section{Acknowledgments}

This work is supported by a Grant for Medical Scientific Research from the health bureau of Guangdong province, R. P. China. (No. A2002298).

\section{Reference}

Barks JD, Silverstein FS. 1992. Excitatory amino acids contribute to the pathogenesis of perinatal hypoxic-ischemic brain injury. Brain Pathol, 2:235-43.

Calabresi P, Centonze D, Cupini LM, et al. 2003. Ionotropic glutamate receptors: still a target for neuroprotection in brain ischemia? Insights from in vitro studies. Neurobiol Dis, 12:82-8.

Calabresi P, Marti M, Picconi B, et al. 2003. Lamotrigine and remacemide protect striatal neurons against in vitro ischemia: an electrophysiological study. Exp Neurol, 182:461-9.

Calabresi P, Picconi B, Saulle E, et al. 2000. Is pharmacological neuroprotection dependent on reduced glutamate release? Stroke, 31:766-73.

Castel-Branco MM, Falcão AC, Figueiredo IV, et al. 2005. Lamotrigine pharmacokinetic/pharmacodynamic modelling in rats. Fundam Clin Pharmacol, 19:669-75.

Chen SD, Lee JM, Yang DI, et al. 2002. Combination therapy for ischemic stroke: potential of neuroprotectants plus thrombolytics. Am J Cardiovasc Drugs, 2:303-13.

Cheung H, Kamp D, Harris E. 1992 . An in vitro investigation of the action of lamotrigine on neuronal voltage-activated sodium channels. Epilepsy Res, 13:107-12.

Crumrine RC, Bergstrand K, Cooper AT, et al. 1997. Lamotrigine protects hippocampal CA1 neurons from ischemic damage after cardiac arrest. Stroke, 28:2230-7.

Graham SH, Chen J, Sharp FR, et al. 1993. Limiting ischemic injury by inhibition of excitatory amino acid release. J Cereb Blood Flow Metab, 13:88-97.

Hattori H, Wasterlain CG.. 1990.Excitatory amino acids in the developing brain: ontogeny, plasticity, and excitotoxicity. Pediatr Neurol, 6:219-28.

Lee WT, Shen YZ, Chang C. 2000. Neuroprotective effect of lamotrigine and MK-801 on rat brain lesions induced by 3-nitropropionic acid: evaluation by magnetic resonance imaging and in vivo proton magnetic resonance spectroscopy. Neuroscience, 95:89-95.

Leach MJ, Marden CM, Miller AA. 1986. Pharmacological studies on lamotrigine, a novel potential antiepileptic drug: II. Neurochemical studies on the mechanism of action. Epilepsia, 27:490-7.

Lees G, Leach MJ. 1993. Studies on the mechanism of action of the novel anticonvulsant lamotrigine (Lamictal) using primary neurological cultures from rat cortex. Brain Res, 612:190-9.
Martens P, Raabe A, Johnsson P. 1998. Serum S-100 and neuron-specific enolase for prediction of regaining consciousness after global cerebral ischemia. Stroke, 29:2363-6.

Mikati MA, Fayad M, Koleilat M, et al. 2002. Efficacy, tolerability, and kinetics of lamotrigine in infants. J Pediatr, 141:31-5.

Nellgård B, Wieloch T. 1992. Postischemic blockade of AMPA but not NMDA receptors mitigates neuronal damage in the rat brain following transient severe cerebral ischemia. J Cereb Blood Flow Metab, 12:2-11.

Ohman I, Vitols S, Tomson T. 2000. Lamotrigine in pregnancy: pharmacokinetics during delivery, in the neonate, and during lactation. Epilepsia, 41:709-13.

Papazisis G, Kallaras K, Kaiki-Astara A, et al. 2007. Neuroprotection by lamotrigine in a rat model of neonatal hypoxic-ischaemic encephalopathy. Int $J$ Neuropsychopharmacol, 26:1-9.

Puka-Sundvall M, Sandberg M, Hagberg H. 1997. Brain injury after hypoxia-ischemia in newborn rats: relationship to extracellular levels of excitatory amino acids and cysteine. Brain Res, 750:325-8.

Rice JE 3rd, Vannucci RC, Brierley JB. 1981. The influence of immaturity on hypoxic-ischemic brain damage in the rat. Ann Neurol, 9:131-41.

Scatton B, Frost J, George P, et al. 1994. Present developments in NMDA receptor antagonists against cerebral ischaemia. Current Patents Lad, 0926-2594:523-545.

Schoerkhuber W, Kittler H, Sterz F, et al. 1999. Time course of serum neuron-specific enolase a predictor of neurological outcome in patients resuscitated from cardiac arrest. Stroke, 30:1598-603.

Schulz JB, Matthews RT, Jenkins BG, et al. 1995. Improved therapeutic window for treatment of histotoxic hypoxia with a free radical spin trap. J Cereb Blood Flow Metab, 15:948-52.

Smith SE, Meldrum BS. 1995. Cerebroprotective effect of lamotrigine after focal ischemia in rats. Stroke, 26:117-22.

Strijbos PJ, Leach MJ, Garthwaite J. 1996. Vicious cycle involving Na+ channels, glutamate release, and NMDA receptors mediates delayed neurodegeneration through nitric oxide formation. J Neurosci, 16:5004-13.

Verdú Pérez A, Falero MP, Arroyos A, et al. 2001. Blood neuronal specific enolase in newborns with perinatal asphyxia. Rev Neurol, 32:714-17.

Wang SJ, Sihra TS, Gean PW. 2001. Lamotrigine inhibition of glutamate release from isolated cerebrocortical nerve terminals (synaptosomes) by suppression of voltage-activated calcium channel activity. Neuroreport, 12:2255-8.

Wang XH, Qing M, Fang SZ. 1999. A study on the changes of S-100, CKBB, NSE and MBP levels in the blood and the cerebrospinal fluid after hypoxic-ischemic brain damage in rats. Chin J Pediatr, 37:670-2.

Walker MC, Tong X, Perry H, et al. 2000. Comparison of serum, cerebrospinal fluid and brain extracellular fluid pharmacokinetics of lamotrigine. Br J Pharmacol, 130:242-8.

Wiard RP, Dickerson MC, Beek O, et al. 1995. Neuroprotective properties of the novel antiepileptic lamotrigine in a gerbil model of global cerebral ischemia. Stroke, 26:466-72.

Yi YH, Guo WC, Liao WP. 2003. Neuroprotective effects of lamotrigine on hypoxic-ischemic encephalopathy in neonatal rats: Relationships with administration time. Epilepsia, 44(s8):177. 
